\title{
Tripchlorolide induces cell death in lung cancer cells by autophagy
}

\author{
LIMIN CHEN, QING LIU, ZHENGHUI HUANG, FENG WU, ZHIYING LI, XIANGQI CHEN and TINGYAN LIN \\ Department of Respiratory Medicine, Union Hospital, Fujian Medical University, Fuzhou, Fujian 350001, P.R. China
}

Received August 13,2011; Accepted November 8, 2011

DOI: 10.3892/ijo.2011.1278

\begin{abstract}
It has been demonstrated that triptolide inhibits the growth of several types of cancer cells in vitro and prevents tumor growth in vivo by inducing apoptosis and autophagy. Here we showed that Tripchlorolide (T4) significantly suppressed the proliferation of A549 cells in a dose- and time-dependent manner. This suppressive effect was diminished when cells were pretreated with 3-Methylamphetamine (3-MA). After the cells were treated with T4, the LC3 II protein expression was significantly increased, and autophagosomes were observed by TEM. However, almost no apoptosis was observed in A549 treated with T4. These results suggest that T4 induces A549 cell death predominantly through the activation of the autophagy pathway instead of the apoptosis pathway.
\end{abstract}

\section{Introduction}

Lung cancer is one of the leading causes of cancer death. The overall 5-year survival rate for lung cancer is less than $15 \%$ (1), principally due to the metastasis, radiation resistance and drug-resistance in the advanced stage. The main reason for drug treatment failure is the disturbance of the regulation of cell death (2).

Apoptosis is the primary process of cell death that is under complex molecular control and can be triggered by various signals. It is well-documented that apoptosis deficiency in lung cancer results in the drug-resistance (3). Therefore, it is priority for lung cancer treatment to find an alternative process that induces lung cancer death. Recently, many more scientists became interested in autophagy, a cellular process called type II cell death. Central to the process is the formation of autophagosomes, double-membrane vesicles that can be observed by transmission electron microscope (TEM) (4-6). Already in 1980 , it was shown that the autophagy capacity of lung cancer is lower than that of surrounding normal cells (7). Furthermore, nutrient deficiency and high cell density increase autophagy

Correspondence to: Dr Ting-Yan Lin, Department of Respiratory Medicine, Union Hospital, Fujian Medical University, Fuzhou, Fujian 350001, P.R. China

E-mail: tingyanlin@yahoo.com

Key words: tripchlorolide, autophagy, A549, lung cancer, apoptosis, LC3 capacity (8). Therefore, the decrease of autophagy capacity may be in favor of cancer survival.

The extract from Tripterygium wilfordii Hook $F$, which has been shown to have strong immune inhibition and antiinflammation activities, is widely used in China to treat certain autoimmune diseases (such as rheumatoid arthritis and kidney diseases) (9). Tripchlorolide (T4) can be extracted from Tripterygium wilfordii Hook $F$, or synthesized from its precursor triptolide after hydroxyl acylation and subsequently chlorination (10). After structure modification, T4 was shown to have similar drug activities, but low toxicities compared to triptolide (11). In the present study, we examined the role of T4 in regulating proliferation, apoptosis and autophagy in lung cancer A549.

\section{Materials and methods}

Materials. Materials used in this study include 3-Methylamphetamine (3-MA) and PI was purchased from Sigma, anti-MAP-LC3-II from Santa Cruz, IgG-FITC from LingFei, goat anti-rabbit secondary antibody from KPL, $\beta$-actin antibody from NeoMarkers, chemiluminescent substrate from KPL, Alex488-conjugated secondary antibody and Topro-3 from Invitrogen.

Cell culture. Lung cancer cell line A549 was obtained from Cell Line Bank, Chinese Academy of Sciences, and maintained in F-12K supplemented with $10 \%$ FBA and $100 \mu \mathrm{g} / \mathrm{ml}$ penicillin and streptomycin at $37^{\circ} \mathrm{C}$ and $5 \% \mathrm{CO}_{2}$. Every two days, cell growth media was replaced with fresh media.

Drug treatment. A549 cells (around 80\% confluence) were treated with/without drugs as below: i) control, cells were not treated with drugs; ii) T4 treatment, cells were exposed to T4 as the final concentrations of $5 \mathrm{nM}, 25 \mathrm{nM}, 50 \mathrm{nM}, 100 \mathrm{nM}$ and $200 \mathrm{nM}$, respectively; iii) 3-MA pretreatment plus T4 treatment, cells were pretreated with $5 \mathrm{mM}$ (final concentration) 3-MA for $1 \mathrm{~h}$, and followed by T4 treatment with various concentrations as indicated above. Cells were collected for the following experiments after they had been treated with/without drugs for $24 \mathrm{~h}$, $48 \mathrm{~h}$ or $72 \mathrm{~h}$. Each experiment was performed in triplicates.

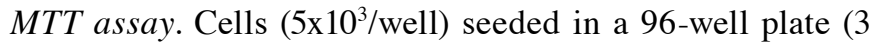
wells per concentration) were exposed to a series of concentrations of T4. The control was supplemented with the same volume of media. Cells were cultured for 0-72 h. MTT (100 $\mu \mathrm{l})$ $(5 \mathrm{~g} / \mathrm{l})$ was added to all the wells and the plate was re-incubated at $37^{\circ} \mathrm{C}$ for $4 \mathrm{~h}$. To each well $90 \mu \mathrm{l}$ of $10 \%$ acid-isopropanol 
solution was added. After $12 \mathrm{~h}$, the plates were read using a test wavelength of $550 \mathrm{~nm}$ with a reference wavelength of 630 $\mathrm{nm}$. The mean OD values of the experiments after calibration were used for calculating the viability rate and the death rate: The viability rate $=\mathrm{A} 550$ sample group/A550 control group, the death rate $=1-$ the viability rate.

Immunofluorescence assay (IFA). A549 cells were washed with $0.01 \mathrm{M}$ PBS, fixed in 4\% paraformaldehyde solution for $30 \mathrm{~min}$ and washed again with PBS. Then A549 were permeabilized by $0.3 \%$ Triton X-100 for 10 min and blocked in TBS (5\% BSA) for $60 \mathrm{~min}$. After adding anti-MAP1-LC3-II (diluted 1:100), the slides were incubated in a moist chamber at $4^{\circ} \mathrm{C}$ overnight and subsequently washed again with PBS. The slides were further incubated with Alex 488-labeled secondary antibodies for $1 \mathrm{~h}$ in a moist chamber at room temperature and washed again. Topro-3 (diluted 1:100,000 in PBS) was added for nuclear staining and incubated for $10 \mathrm{~min}$, the slides were washed with PBS, and anti-fade mounting medium was added, then covered with cover slip, sealed with nail polish, and examined by fluorescence microscopy. Micrographs were taken for result assessment. The experiment design included negative control and blank control.

The examination of autophagosome by TEM. Cells were fixed in $3 \%$ glutaradehyde $1.5 \%$ paraformaldehyde for days or hours at $4{ }^{\circ} \mathrm{C}$, and postfixed with $1 \% \mathrm{OsO}_{4} / 1.5 \%$ potassiumferrocyanide for $1.5 \mathrm{~h}$. After washing with PBS, cells were stained with $70 \%$ ethanol saturated with uranyl acetate, followed by gradient dehydration with ethanol-acetone, and finally embedded in epoxy resin 618 for section. The ultrathin sections $(50 \mathrm{~nm})$ were stained by uranyl acetate and lead citrate for $5 \mathrm{~min}$, respectively, and examined and photographed with TEM.

Western blotting. Cells were lysed by lysate buffer $(10 \mathrm{mmol} / \mathrm{l}$ Tris, pH 7.4, $100 \mathrm{mmol} / 1 \mathrm{NaCl}, 1 \mathrm{mmol} / \mathrm{l}$ EDTA, $1 \mathrm{mmol} / \mathrm{l}$ EGTA, $1 \mathrm{mmol} / 1 \mathrm{NaF}, 20 \mathrm{mmol} / 1 \mathrm{Na}_{4} \mathrm{P}_{2} \mathrm{O}_{7}, 2 \mathrm{mmol} / 1 \mathrm{Na}_{3} \mathrm{VO}_{4}$, $0.1 \%$ SDS, $0.5 \%$ sodium deoxycholate, $1 \%$ Triton-X 100 , $1 \mathrm{mmol} / 1 \mathrm{PMSF}, 60 \mu \mathrm{g} / \mathrm{ml}$ aprotinin, $10 \mu \mathrm{g} / \mathrm{ml}$ leupeptin, $1 \mu \mathrm{g} / \mathrm{ml}$ pepstatin) on ice for $30 \mathrm{~min}$, centrifuged at 12,000 g for $30 \mathrm{~min}$, and collected the supernatant. The supernatant was diluted to $2 \mathrm{mg} / \mathrm{ml}$ (protein concentration), mixed 1:1 with loading buffer (62.5 mM Tris-HCl, pH 6.8, $10 \%$ glycerol, $2 \%$ SDS, and $0.1 \%$ bromophenol blue), and boiled for $5 \mathrm{~min}$. After cooling down, samples $(30 \mu \mathrm{g} /$ lane) were loaded and run on a SDS-PAGE (10\%) gel. The separated proteins were transferred to PVDF membrane. Membrane was blocked in TBS supplemented with $5 \%$ non-fat milk and $0.2 \%$ Tween-20 for $1 \mathrm{~h}$, and incubated with primary antibody (diluted 1:500) at $4^{\circ} \mathrm{C}$ overnight. Following incubation with secondary antibody (coat anti-rabbit HRPO, diluted 1:4,000) for $2 \mathrm{~h}$ the membrane was exposed to film. The images of bands were assessed by software from Scion Co. $\beta$-actin was used as loading control.

Statistical analysis. Data are expressed as the mean \pm SD (standard deviation) of triplicates. The significance of the differences among the treatments was assessed by one-way analysis of variance (ANOVA). All analyses were conducted with SPSS statistical package. $\mathrm{P}<0.05$ was considered statistically significant.

\section{Results}

The toxicity of T4. A549 cells were treated with a series of concentrations of T4 (0-200 nM) for different periods (24-72 h). The viability of A549 was reduced with increasing T4 concentration and longer incubation time (Fig. 1). After exposure to $200 \mathrm{nM}$ T4 for $24 \mathrm{~h}$, the cells viability declined to around $50 \%$. These results suggested that $\mathrm{T} 4$ suppressed the proliferation of A549 in a dose- and time-dependent manner. When the cells were pretreated with 3-MA, the inhibitor of autophagy, T4 toxicity was dramatically reduced. Compared to the controls, the 3-MA pretreated cells followed by T4 treatment were not shown to have significance difference of the cell viability, except those exposed to $100 \mathrm{nM}$ T4 for $48-72 \mathrm{~h}$ or $200 \mathrm{nM}$ T4 for 24-72 h.

T4 rapidly induced $A 549$ autophagy. Because the $\mathrm{IC}_{50}$ for $\mathrm{T} 4$ is $200 \mathrm{nM}$ and longer incubation periods dramatically increase the number of dead cells, we chose $200 \mathrm{nM}$ T4 for $24 \mathrm{~h}$ treatment as the sample-conditions of IFA. In the control, the results of IFA showed that LC3 (microtubule-associated protein 1 light chain 3) was expressed at low level and distributed in the cytoplasm (Fig. 2). In cells treated with $200 \mathrm{nM}$ T4 for $24 \mathrm{~h}$, the protein expression level of LC3 was dramatically upregulated, and intense granular staining of LC3 was observed. However, in the cells pretreated with 3-MA, LC3 protein level was similar to that in the control. It indicated that T4 can induce A549 autophagy.

The effect of T4 on apoptosis. To determine whether T4 induced A549 apoptosis or not, we examined the apoptotic bodies in the cells exposed to $200 \mathrm{nM}$ T4 for $24 \mathrm{~h}$. In the treated cells, no typical apoptotic bodies were observed, as revealed by the TUNEL assay (Fig. 3). Therefore, A549 death induced by T4 was not through apoptosis pathway.

T4 induced autophagosome formation in A549. To further confirm that T4 can induce A549 autophagy, we checked the formation of autophagosomes in T4 treated cells using TEM. In the control, many normal vesicles containing subcellular organelles were observed (Fig. 4). In the T4 treated cells, damaged organelles were observed, such as swollen mitochondria surrrounded by double-membrane vacuoles, which further formed autophagosomes. Autophagosome subsequently fused with a lysozome and the internal material was degraded. Undegraded debris within the autolysosomes was also observed.

T4 stimulated LC3 expression in A549 cancer cell. LC3 is proposed to serve as a marker of autophagosome membrane (16). As determined by Western blotting, the protein level of LC3 II was upregulated with the increase of T4 concentration, and reached the peak at $200 \mathrm{nM}$ T4 treatment (Fig. 5). However, in the 3-MA pretreated cells followed by T4 treatment, the level of LC3 II was much lower than that in the cells only treated by T4, though it was slightly higher than that in the control. These results suggested that T4 stimulated LC3 II expression of A549, while 3-MA weakened the effect. Thus, it was further confirmed that T4 induced cell death in A549 by autophagy. 
$\mathbf{A}$

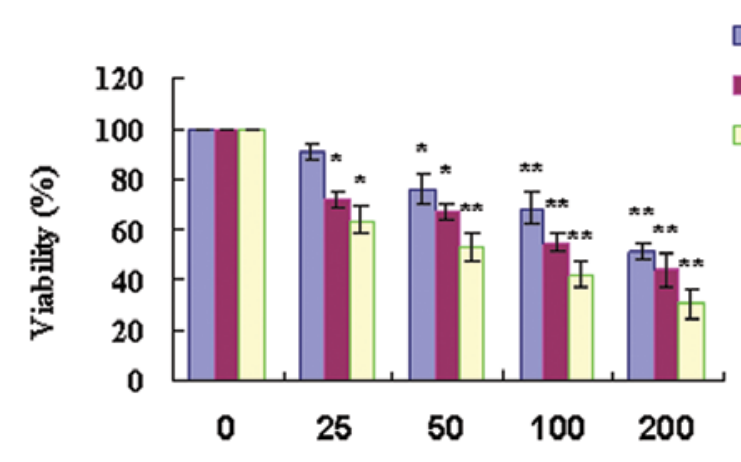

$\mathrm{T} 4(\mathrm{nM} / \mathrm{L})$
B

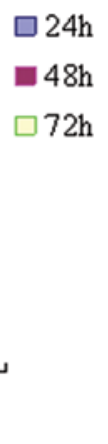

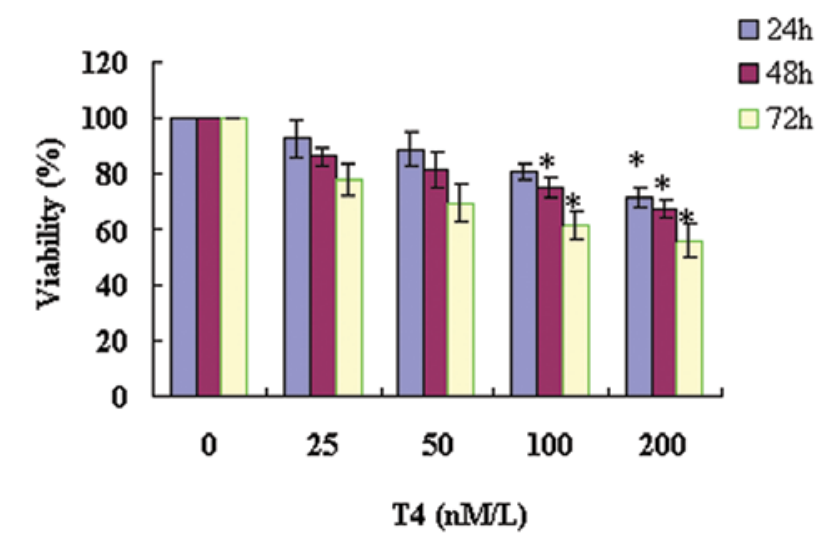

Figure 1. The toxicity of T4. (A) T4 treatment and (B) 3-MA pretreatment followed by $\mathrm{T} 4$. $\left({ }^{*} \mathrm{P}<0.05,{ }^{\text {*** }} \mathrm{P}<0.01\right)$.

A

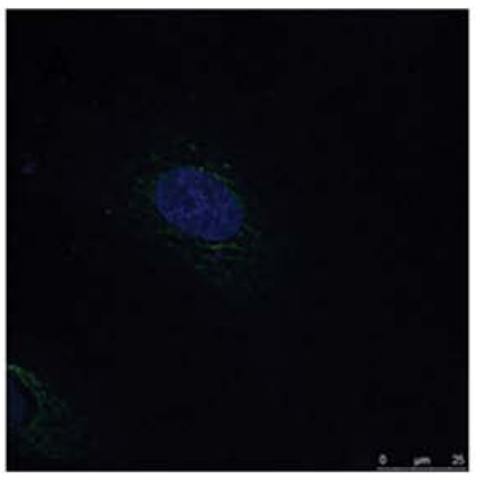

B

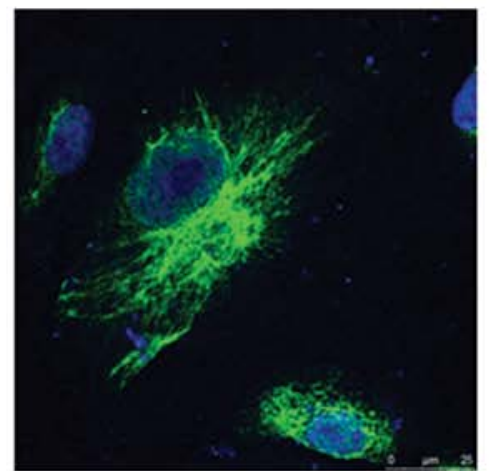

$\mathrm{C}$

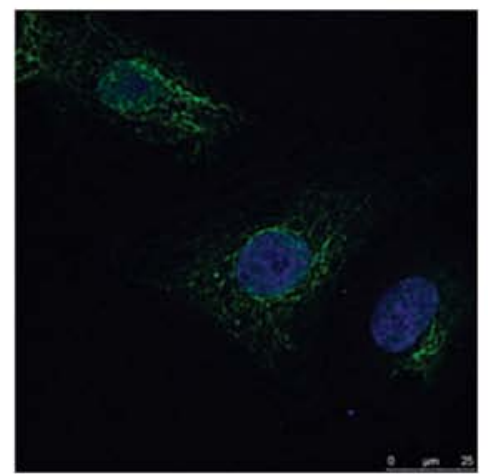

Figure 2. T4 induced A549 autophagy. (A) control. (B) T4 treatment, positive bodies labeled by LC3 increased dramatically (200 nM T4 treatment for $24 \mathrm{~h}$ ). (C) 3-MA +T4 treatment, LC3 level was decreased compared to the level in the control cells.

A

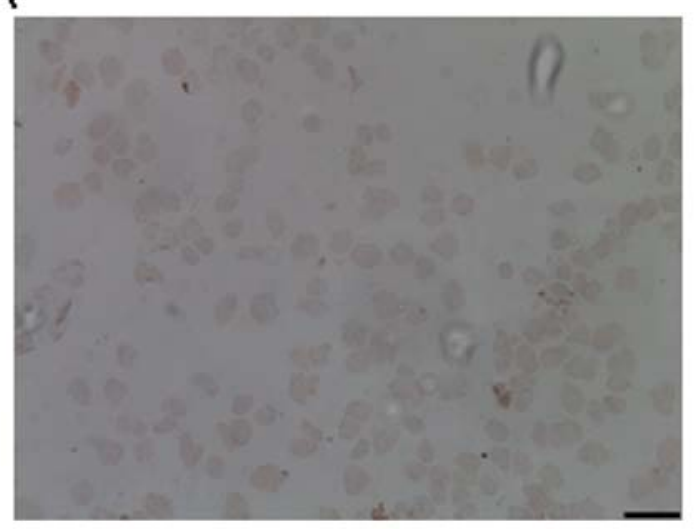

B

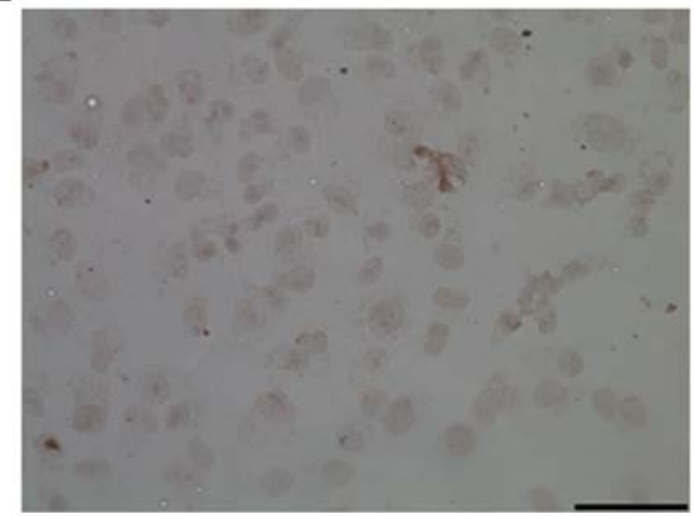

Figure 3. The effect of T4 on A549 apoptosis. (A) Control. (B) Treatment: no obvious positive apoptotic bodies were observed in the cells treated by T4.

\section{Discussion}

Autophagy, the type II cell death, is different from apoptosis (type I cell death). Autophagy is a strictly controlled process in regulating cellular contents degradation and recycle, and plays an essential role in organelles metabolism and bioenergy supplementation. Malfunction of autophagy has been associated with many human diseases, such as cancer and 
A

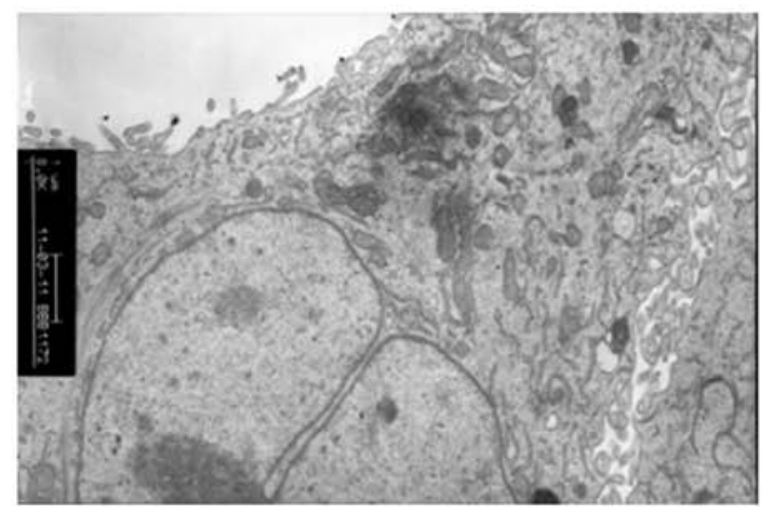

$\mathrm{B}$

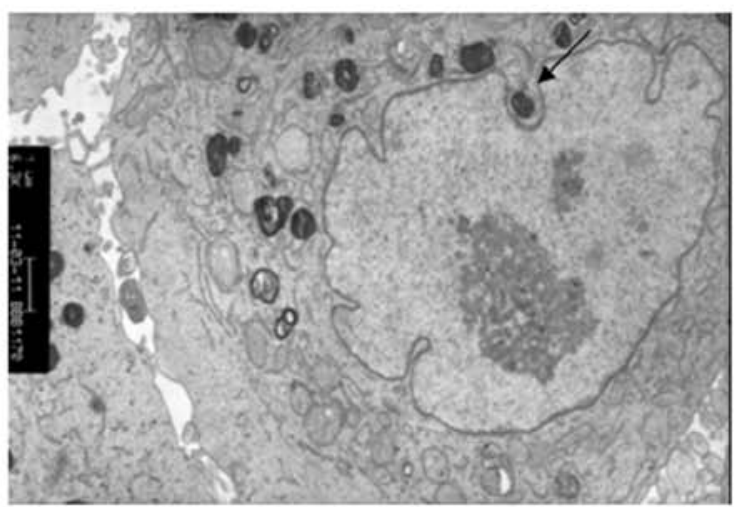

Figure 4. The formation of autophagosomes in A549. (A) control. (B) T4 treated cells: autophagosomes were observed.

A
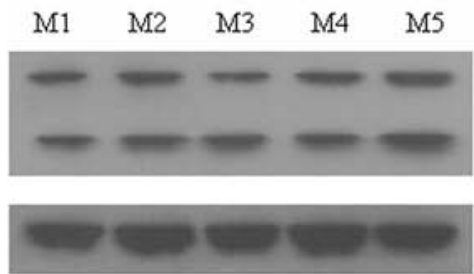

$\mathrm{B}$
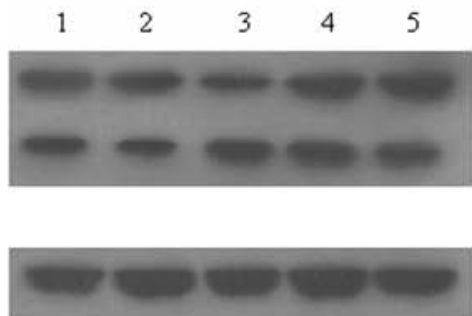

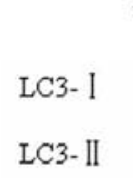

LC3- I

LC3-II

actin
C

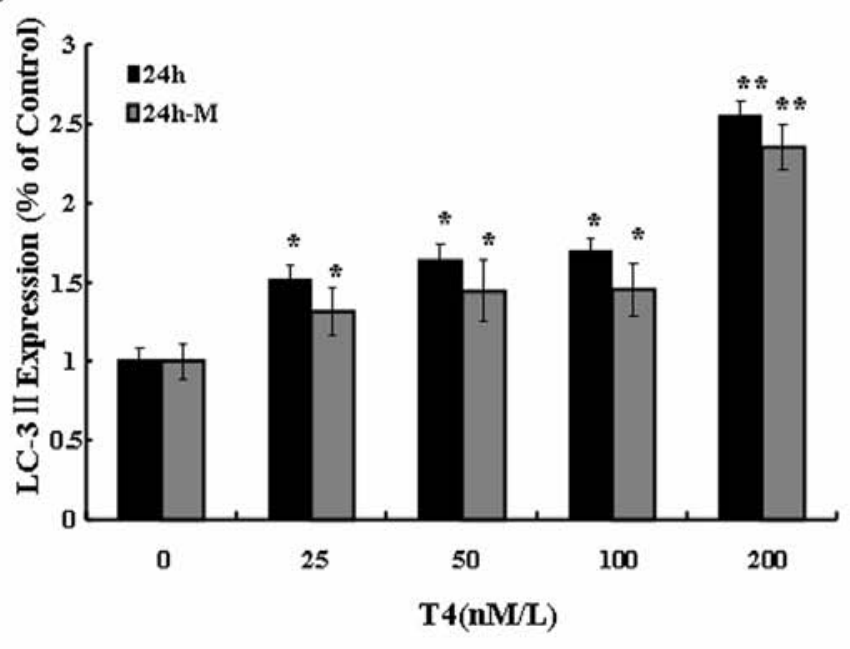

Figure 5. T4 upregulated LC3 expression in A549. 1-5: T4 concentration: 0, $25 \mathrm{nM}, 50 \mathrm{nM}, 100 \mathrm{nM}$ and $200 \mathrm{nM}$, respectively. M1-M5: cells were pretreated with 3-MA, T4 concentrations: $0,25 \mathrm{nM}, 50 \mathrm{nM}, 100 \mathrm{nM}$ and $200 \mathrm{nM}$, respectively. (A) T4 treatment; (B) pretreated with 3-MA and followed by T4; (C) T4 treatment significantly upregulated the level of LC3-II in A549.

neurodegene-rative disorders (12). Cancer is one of the earliest diseases found to be related to autophagy (13). Autophagy inhibits tumorigenesis mainly through regulation of the concentration of cellular peroxide, diminishing the disturbance of protein metabolism and maintenance of cell homeostasis. The decrease of autophagy activity promotes oxidation stress and thereby increases accumulation of cancer-related mutants (14).

Autophagic activity of cancer cells is lower than that of normal cells (7). The decrease of autophagic capacity is also found in precancerous cells of some cancers, such as hepatocarcinoma induced by chemical carcinogen. Reduced autophagic capacity is in favor of carcinomagenesis. It is necessary for cells to maintain a normal acutophgic capacity for cleaning the damaged organelles due to chemical carcinogens, radioation and oxidation stress, and thus maintaining genome integrity and reducing tumor occurrence. Secondly, autophagy degrades organelles (such as endoplasmic reticulum and Golgi) and longlived protein, and thereof results in negative protein balance in premalignant cells, inhibiting uncontrolled proliferation (15). In addition, hyperactive autophagy induces tumor cell death (16-18).

Recently, it has been reported that some herbal drugs were used for cancer treatment due to their effect on cell autophagy. For exempt, Aloe-emodin, a herbal anthraquinone derivative, induced rat C6 glioma autophagic death (19). Reseratrol, a natural phytoalexin present in grapes, nuts and red wine, induced ovarian cancer cell death through autophagy (20). 6-Shogaol, an active component from ginger, induced A549 autophagy by suppressing the AKT/mTOR pathway (21). Triptolide, the precursor of T4, inhibited the growth of hamster cholangiocarcinoma (22) and human tumors transplanted into nude mice (23). Several studies revealed that triptolide suppressed the growth of pancreatic cancer and neuroblastoma both in vivo and in vitro $(24,25)$. Nammeeta et al found that triptolide induced pancreatic cancer cell death through apoptosis and autophagy (26). Furthermore, triptolide in combination with TRAIL significantly decreased pancreatic cancer cell viability (27). However, the role 
of triptolide in regulating lung cancer cells has not been reported. The clinical application of the crude extract from Tripterygium wilfordii is restricted due to its high toxicity.

In the present study, we studied the role of T4 in A549 lung cancer cells. MTT results showed that T4 induced cytoxity in A549 cell death in a dose- and time-dependent manner. Autophagosomes were observed by TEM in A549 treated by T4. When A549 was pretreated with 3-MA and followed by T4 treatment, the mortality of A549 was much lower than that in A549 only exposed to T4. Although apoptosis adduced by triptolide was found in various cancer cells $(26,28-30)$, almost no apoptosis was observed in A549 treated T4. Therefore, T4 suppressed the proliferation of A549 principally through activating autophagy pathway, but not apoptosis pathway. A recent study showed that triptolide promotes lung cancer apoptosis dependent on TRAIL (31). Further studies are required to investigate the effect of T4 in combination with TRAIL on lung cancer cells.

Autophagosome formation is known to have a central role in autophagy. Two ubiquitin-like conjugation systems are essential for the formation. One is Atg12-Atg5 conjugation system, and the other is Atg8 regulated lipidation system (15). LC3, the mammalian homologue of Atg8, has three subtypes: pre-LC3, LC3-I and LC3-II. pre-LC3 is processed by the cleavage of a portion (22 amino acids) of its C-terminal to form LC3-I, which is distributed in the cytoplasm. LC3-I is converted to LC3-II by further cleavage and conjugation to phosphatidylethanolamine. LC3-II is recruited on the membrane of pre-autophagosome by Atg 5 and still located on autophagosome membrane after autophagosome formation. Therefore, LC3-II is proposed to serve as a marker of cell autophagy (15). IFA results showed that the number of LC3-positive granules was significantly increased in A549 treated by T4. However, in the 3-MA pretreated cells, followed by $\mathrm{T} 4$ treatment, autophagosomes were dramatically reduced compared to the control. Moreover, the protein level of LC3-II was upregulated after T4 treatment in a time- and concentration-dependent manner, and reached the peak at 200 nM. 3-MA pretreatment dramatically reduced the protein level of LC3-II in A549, though it was slightly higher than that of the control. These results indicate that T4 kills lung cancer cells by promoting autophagy, which is different from the mechanism of anti-cancer effect of triptolide for other cancers (26,28-30).

In conclusion, our study confirmed that $\mathrm{T} 4$ suppressed the proliferation of A549 cells by activating autophagy. The study provides new evidence for T4 clinical application in lung cancer treatment.

\section{Acknowledgements}

We thank H.J. Ren for critical reading of the manuscript. This study was supported by Education Department of Fujian Province (JA10142).

\section{References}

1. Gilligan D, Nicolson M, Smith I, et al: Preoperative chemotherapy in patients with resectable non-small cell lung cancer: results of the MRC LU22/NVALT 2/EORTC 08012 multicentre randomised trial and update of systematic review. Lancet 369: 1929-1937, 2007.

2. Melet A, Song K, Bucur O, Jagani Z, Grassian AR and Khosravi-Far R: Apoptotic pathways in tumor progression and therapy. Adv Exp Med Biol 615: 47-79, 2008.

3. Gozuacik D and Kimchi A: Autophagy as a cell death and tumor suppressor mechanism. Oncogene 23: 2891-2906, 2004.
4. Edinger AL and Thompson CB: Death by design: apoptosis, necrosis and autophagy. Curr Opin Cell Biol 16: 663-669, 2004.

5. Boya P, Gonzalez-Polo RA, Casares N, et al: Inhibition of macroautophagy triggers apoptosis. Mol Cell Biol 25: 1025-1040, 2005.

6. Cuervo AM: Autophagy: in sickness and in health. Trends Cell Biol 14: 70-77, 2004.

7. Cuervo AM and Dice JF: A receptor for the selective uptake and degradation of proteins by lysosomes. Science 273: 501-503, 1996.

8. Ogier-Denis E and Codogno P: Autophagy: a barrier or an adaptive response to cancer. Biochim Biophys Acta 1603: 113-128, 2003.

9. Messina ME Jr and Halaby R: Does triptolide induce lysosomalmediated apoptosis in human breast cancer cells? Med Hypotheses pp9193, 2011.

10. Yang GZ and Chen Y: The study development of ester ethanol of common threewingnut root. J Chin Med Mater 29: 200-203, 2006

11. Pan XD, Chen XC,Zhu YG, et al: Tripchlorolide protects neuronal cells from microglia-mediated beta-amyloid neurotoxicity through inhibiting NF-kappaB and JNK signaling. Glia 57: 1227-1238, 2009.

12. Klionsky DJ and Emr SD: Autophagy as a regulated pathway of cellular degradation. Science 290: 1717-1721, 2000.

13. Levine B and Kroemer G: Autophagy in the pathogenesis of disease. Cell 132: 27-42, 2008.

14. Holm E, Hildebrandt W, Kinscherf R and Droge W: Low postabsorptive net protein degradation in male cancer patients: lack of sensitivity to regulatory amino acids? Oncol Rep 17: 695-700, 2007.

15. Hait WN, Jin S and Yang JM: A matter of life or death (or both): understanding autophagy in cancer. Clin Cancer Res 12: 1961-1965, 2006.

16. Qu X, Yu J, Bhagat G, et al: Promotion of tumorigenesis by heterozygous disruption of the beclin 1 autophagy gene. J Clin Invest 112: 1809-1820, 2003.

17. Qian W, Liu J, Jin J, Ni W and Xu W: Arsenic trioxide induces not only apoptosis but also autophagic cell death in leukemia cell lines via up-regulation of Beclin-1. Leuk Res 31: 329-339, 2007.

18. Kim KW, Mutter RW, Cao C, et al: Autophagy for cancer therapy through inhibition of pro-apoptotic proteins and mammalian target of rapamycin signaling. J Biol Chem 281: 36883-36890, 2006.

19. Mijatovic S, Maksimovic-Ivanic D, Radovic J, et al: Anti-glioma action of aloe emodin: the role of ERK inhibition. Cell Mol Life Sci 62: 589-598, 2005.

20. Opipari AW Jr, Tan L, Boitano AE, Sorenson DR, Aurora A and Liu JR: Resveratrol-induced autophagocytosis in ovarian cancer cells. Cancer Res 64: 696-703, 2004.

21. Hung JY, Hsu YL, Li CT, et al: 6-Shogaol, an active constituent of dietary ginger, induces autophagy by inhibiting the AKT/mTOR pathway in human non-small cell lung cancer A549 cells. J Agric Food Chem 57: 9809-9816, 2009.

22. Tengchaisri T, Chawengkirttikul R, Rachaphaew N, Reutrakul V, Sangsuwan R and Sirisinha S: Antitumor activity of triptolide against cholangiocarcinoma growth in vitro and in hamsters. Cancer Lett 133: 169-175, 1998.

23. Yang S, Chen J, Guo Z, et al: Triptolide inhibits the growth and metastasis of solid tumors. Mol Cancer Ther 2: 65-72, 2003.

24. Phillips PA, Dudeja V, McCarroll JA, et al: Triptolide induces pancreatic cancer cell death via inhibition of heat shock protein 70. Cancer Res 67: 9407-9416, 2007.

25. Antonoff MB, Chugh R, Borja-Cacho D, et al: Triptolide therapy for neuroblastoma decreases cell viability in vitro and inhibits tumor growth in vivo. Surgery 146: 282-290, 2009.

26. Mujumdar N, Mackenzie TN, Dudeja V, et al: Triptolide induces cell death in pancreatic cancer cells by apoptotic and autophagic pathways. Gastroenterology 139: 598-608, 2010.

27. Borja-Cacho D, Yokoyama Y, Chugh RK, et al: TRAIL and triptolide: an effective combination that induces apoptosis in pancreatic cancer cells. J Gastrointest Surg 14: 252-260, 2010.

28. Meng HT, Zhu L, Ni WM, You LS, Jin J and Qian WB: Triptolide inhibits the proliferation of cells from lymphocytic leukemic cell lines in association with downregulation of NF-kappaB activity and miR-16-1*. Acta Pharmacol Sin 32: 503-511, 2011.

29. Li J, Zhu W, Leng T, et al: Triptolide-induced cell cycle arrest and apoptosis in human renal cell carcinoma cells. Oncol Rep 25: 979-987, 2011.

30. Wu PP, Liu KC, Huang WW, et al: Triptolide induces apoptosis in human adrenal cancer NCI-H295 cells through a mitochondrialdependent pathway. Oncol Rep 25: 551-557, 2011

31. Lee KY, Park JS, Jee YK and Rosen GD: Triptolide sensitizes lung cancer cells to TNF-related apoptosis-inducing ligand (TRAIL)induced apoptosis by inhibition of NF-kappaB activation. Exp Mol Med 34: 462-468, 2002. 